\title{
¿Estaría indicada la heparina para prevenir trombosis venosa profunda en pacientes inmovilizados por lesiones menores en miembros inferiores?
}

\author{
B. N. Romera Pintor, M. J. Flores Ugart, M. T. M ombiela Muruzabal* \\ C. González Manjavacas*, S. Álvarez Montero** \\ MIR de tercer año de Medicina Familiar y Comunitaria. *Tutor de MIR de M FyC. \\ C.S. Monterrozas. Madrid. $* *$ Tutor MIR de MFyC. C.S. de Torrelodones. Madrid
}

\section{RESUMEN}

La profilaxis con heparina de bajo peso molecu lar (HBPM) es eficaz para reducir el riesgo de trombosis venosa profunda (TVP) en pacientes es cayolados por traumatismo menor en miembros in feriores. Para prevenir una TVP es necesario tra tar a 23 pacientes (de 13 a 84 para un intervalo de confianza del 95\%) durante aproximadamente 20 días.

Palabras clave: Tromboprofilaxis. Heparina. In movilización. Miembros inferiores.
Should patients with inmovilization of the lower limb be treated with low molecular weight heparin for preventing deep vein thrombosis?

\section{ABSTRACT}

Thromboprophylaxis with low molecular weight heparin (LMWH) is effective in reducing the risk of deep-vein thrombosis (DVT) in patients with plas ter-cast inmovilization of the lower limb. It is necessary to treat 23 patients (CI $95 \%$ : 13 to 84, $p<0,05)$ during about 20 days, for the prevention of one DVT.

Key words: Thromboprophylaxis. Heparin. In movilization. Legs.

\section{ESCENARIO CLÍNICO}

En nuestra práctica diaria vemos cada vez con mayor frecuencia, que los pacientes remitidos desde un centro hospitalario para control por su médico de una lesión menor de miembros inferiores en tratamiento con escayola, están siendo tratados además con profilaxis antitrombótica. Esto nos ha llevado a plantearnos la eficacia de este tipo de intervención.

\section{CUESTIÓN ESTRUCTURADA}

¿En pacientes con lesiones menores de miembros inferiores (MMII) que requieren inmoviliza- ción con escayola, es útil el tratamiento preventivo con heparina subcutánea frente a no intervención?

\section{ESTRATEGIA DE BÚSQUEDA}

Nuestra búsqueda tiene como objetivo obtener de la forma más eficiente y rigurosa posible la mejor respuesta disponible para la pregunta planteada. Por eso, a la hora de seleccionar la información renunciamos a priori a intentar hacer una revisión sistemática que nos llevaría un tiempo excesivo, y a combinar datos o realizar cualquier tipo de metaanálisis con los trabajos encontrados (por tratarse de una metodología compleja con la cual no esta-

Aceptación: 25-02-02 
mos familiarizados la mayoría de los médicos de familia). Tratamos, por tanto, de encontrar la mejor publicación disponible: si es una buena revisión sistemática ya hecha por otros, mejor. Si no es así, y surgen varios trabajos que abordan el tema de forma similar, optamos por aquél de mayor calidad metodológica. Los datos del resto podremos utilizarlos, si fuera necesario, como un complemento a la información obtenida con el artículo elegido.

La búsqueda comenzó en la librería Cochrane: http://www.obgyn.net/cochrane.asp sin que se encontraran revisiones sistemáticas.

En Medline el 11/10/2001 se siguió la siguiente estrategia: "Leg injuries"(Mesh) and heparin (Mesh) not "Hip Fractures" (Mesh) Field: All Fields, Limits: 10 Years, only items with abstracts, Human.

Se han revisado 22 títulos. De éstos, se seleccionaron 4 resúmenes, con las siguientes características en común: ensayos clínicos abiertos, prospectivos, aleatorizados, en los que se comparaba la incidencia de Trombosis Venosa Profunda (TVP) en un grupo con profilaxis con Heparina de Bajo Peso Molecular (HBPM) con la de un grupo sin profilaxis. Todos concluían que las HBPM eran eficaces. Se desestimaron tres de ellos porque la muestra que estudiaban era demasiado pequeña, y porque uno de ellos estaba escrito en alemán y publicado en una revista a la que no nos sería fácil acceder. Finalmente se seleccionó el artículo de Kock et al. ${ }^{1}$ porque nos parecía el más riguroso metodológicamente y porque se trata de un ensayo clínico con un diseño idóneo para valorar la eficacia de un tratamiento. La muestra estudiada es amplia, y el tipo de pacientes es similar al que nos encontramos en nuestra práctica en Atención Primaria.

\section{EL ESTUDIO}

Se trata de un ensayo clínico, aleatorizado, abierto, en el que se estudia el efecto de la profilaxis con heparina de bajo peso molecular (HBPM) en la incidencia de trombosis venosa profunda
(TVP), en pacientes con lesiones menores de miembros inferiores, en tratamiento ambulatorio con inmovilización con escayola.

Contexto: pacientes estudiados ambulatoriamente en el Servicio de Traumatología.

Características de los pacientes: Se reclutaron 428 pacientes de los cuales se excluyeron $32 ; 5$ renunciaron a formar parte del estudio; los datos de 52 pacientes no pudieron ser examinados, bien por cambio de grupo, bien porque se decidió tratamiento quirúrgico. Criterios de inclusión: pacientes de edad entre 18-65 años, de ambos sexos, con inmovilización de MMII con escayola. Criterios de exclusión: antecedentes de TVP previa; embarazo; Tratamiento con Anticoagulantes Orales (ACO) o transtornos de la coagulación; puntos de sangrado; insuficiencia venosa crónica; contraindicaciones para profilaxis con heparina; inmovilización con escayola tras tratamiento quirúrgico.

Grupo control: 163 (104 varones), no intervención.

Grupo experimental: 176 (104 varones), heparina de bajo peso molecular subcutánea en una dosis diaria.

\section{RESULTADOS}

En todos lo pacientes se descartó la existencia previa de TVP, con una exploración clínica, medida del perímetro de la pierna, eco-doppler, y realización de flebografía para confirmar cualquier hallazgo sospechoso. Al retirar la escayola, se repitieron estas exploraciones.

En el grupo control hubo 7 TVP. El tiempo medio de inmovilización en ese grupo fue de 18,8 días. En el grupo con profilaxis no hubo ningún caso de TVP. No hubo diferencias significativas en el tiempo de inmovilización entre ambos grupos, ni se desarrollaron complicaciones mayores.

En la tabla I se resumen los datos principales.

En cuanto a la aparición de efectos secundarios, no hubo complicaciones mayores (sangrado, necrosis cutánea, trombocitopenia) en ninguno de los grupos.

\begin{tabular}{|c|c|c|c|c|c|c|}
\hline \multicolumn{7}{|c|}{ RESUMEN DE RESULTADOS } \\
\hline Medición & Tiempo & $\begin{array}{l}\text { Riesgo } \\
\text { grupo } \\
\text { control }\end{array}$ & $\begin{array}{c}\text { Riesgo } \\
\text { grupo } \\
\text { experim. }\end{array}$ & $\begin{array}{l}\text { Reducción } \\
\text { relativa del } \\
\text { riesgo }\end{array}$ & $\begin{array}{c}\text { Reducción } \\
\text { absoluta } \\
\text { del riesgo }\end{array}$ & NNT \\
\hline \multirow{2}{*}{$\begin{array}{l}\text { Trombosis venosa } \\
\text { profunda }\end{array}$} & $\begin{array}{c}18 \text { (2-72) } \\
\text { días }\end{array}$ & 0,043 & 0 & $100 \%$ & 0,043 & 23 \\
\hline & \multicolumn{3}{|c|}{ Intervalos de confianza 95\% } & & $\begin{array}{c}0,012 \text { a } \\
0,074\end{array}$ & 13 a 84 \\
\hline
\end{tabular}




\section{COMENTARIOS}

La profilaxis de accidentes tromboembólicos con el uso de heparinas de bajo peso molecular en pacientes operados se ha convertido en un procedimiento estándar. Hasta ahora, las recomendaciones en las guías de práctica clínica de tromboprofilaxis para pacientes no quirúrgicos se centran en pacientes con riesgo moderado-alto

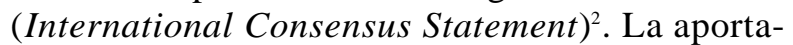
ción del artículo seleccionado es que estudia la incidencia de TVP en pacientes con bajo riesgo (en los que siguiendo las recomendaciones actuales no utilizaríamos la tromboprofilaxis), cuando son tratados con profilaxis frente a no intervención.

\section{-Validez:}

El artículo fue evaluado según los criterios de validez propuestos por Guyatt ${ }^{3}$. Se realizó una asignación aleatoria de pacientes en cada grupo, no hubo diferencias significativas entre los grupos, acabaron el estudio más del $80 \%$ de los pacientes y se hizo un análisis estadístico por intención de tratar (método por el cual todos los pacientes de cada grupo cuentan en el análisis, aunque no hayan completado o recibido el tratamiento, para evitar una exageración de los resultados a favor de la intervención experimental).

Como puntos débiles del estudio hay que señalar que no se especifica el proceso de aleatorización y que la muestra es relativamente pequeña, seleccionada en un centro de traumatología, lo que debilita su aplicabilidad en pacientes tratados desde Atención Primaria. Además, el diseño no es ciego por motivos éticos (hay un riesgo derivado de inyectar un fármaco inocuo) y porque se estimó que el efecto placebo en este caso debería ser irrelevante.

\section{-Importancia:}

La reducción absoluta del riesgo fue del 4,3\% con un intervalo de confianza del $95 \%$ entre el 1,2 y el $7,4 \%$. Con estos resultados, encontramos que para prevenir un caso de TVP sería necesario tratar a 23 pacientes (13-84) durante 20 días.

Estos datos se corroboran (además de en otros dos de los cuatro trabajos encontrados) en otro estudio (el tercero de los cuatro) de similares características (Incidence and Prophylaxis of Deep Venous Thrombosis in Outpatiens with Injury of the Lower Limb $)^{4}$ en el que también se cumplen criterios de validez (estudio de casos y controles, aleatorizado, abierto, con seguimiento de más del $80 \%$ de los pacientes) y en el que la reducción absoluta del riesgo es del $11,8 \%$ (con un intervalo de confianza del $95 \%$ entre 4,3 y $19,2 \%$ ). Aquí el NNT es de 8 (523).
En nuestro estudio, la prevención de un episodio de TVP requiere tratar únicamente a 23 pacientes con una intervención con escasos efectos secundarios, durante el tiempo que dure la inmovilización, lo cual establecería la indicación de profilaxis con HBPM en pacientes de bajo riesgo. Sin embargo, hay que tener en cuenta que se trata de datos referidos a la morbilidad causada por una TVP. La incidencia de tromboembolismo pulmonar fatal se sitúa entre un 0,01 y un $1 \%$ de las TVP, incluso en pacientes con riesgo medio, lo que hace que el impacto de esta intervención sobre la mortalidad por TVP sea muchísimo menor. Además, los intervalos de confianza son relativamente amplios, lo cual resta precisión al resultado.

\section{-Aplicabilidad:}

Los pacientes estudiados y el medio en que se desarrolla el estudio son similares a nuestro medio habitual de práctica clínica. Los pacientes seleccionados presentaban lesiones menores de miembros inferiores, que vemos en Atención Primaria; pero en nuestra experiencia en las consultas de Atención Primaria, gran parte de los traumatismos e inmovilizaciones en MMII que tratamos, se producen en personas jóvenes. En cuanto a la distribución por grupos de edad de la incidencia de TVP, encontramos que de los 163 pacientes que constituyen el grupo control, 44 son > de 40 años, y 119 son $\leq 40$ años.

De los 7 casos de TVP, 5 ocurrieron en el subgrupo control de $>40$ años $(11,4 \%)$, y 2 en el de $\leq 40$ años $(1,7 \%)$. Considerando el tamaño reducido de estos subgrupos, no podemos encontrar evidencia sobre la importancia de la edad como factor de riesgo en este artículo. Sería necesario un estudio con una muestra mayor, donde se analizaran los distintos grupos de edad para estudiar la importancia de este factor.

Las dosis de HBPM empleadas en ambos estudios fueron $32 \mathrm{mg}$ de Mono-Embolex (Sandoz) ${ }^{1} \mathrm{y}$ $36 \mathrm{mg}$ de fraxiparina ${ }^{4}$ respectivamente, mientras que las dosis para profilaxis empleadas habitualmente en nuestro medio son de $20 \mathrm{mg}$ o $40 \mathrm{mg}$ de enoxaparina, que son dosis equivalentes. La relación coste-beneficio parece asumible, aunque no contamos con estudios al respecto.

Estos datos sugieren que las HBPM son eficaces en pacientes no quirúrgicos con una inmovilización con escayola por lesiones menores en miembros inferiores. Sin embargo, las evidencias parecen muy débiles cuando la edad es inferior a 40 años.

Por tanto, concluimos que el uso de férulas para tratar lesiones menores en miembros inferiores debería restringirse en la medida de lo posible, recu- 
rriéndose a otros procedimientos que impliquen una menor inmovilización.

En caso de que haya que colocar una férula de escayola, se debería informar al paciente de la existencia de un riesgo de trombosis venosa profunda (complicación potencialmente grave), que se puede reducir mediante el uso de HBPM.
CORRESPONDENCIA:

Blanca Romera Pintor

Centro de Salud Monterrozas

Avda. Aristóteles s/n

28230 Las Rozas. Madrid

e-mail: blanca_romera@hotmail.com

\section{Bibliografía}

1. Kock HJ, Schmit-Neuerburg KP, Hanke J, Rudofsky G, Hirche H. Thromboprophylaxis with low-molecular-weight heparin in outpatients with plaster-cast immobilisation of the leg. The Lancet 1995; 346: 459-61.

2. Bick RL, Haas SK. International Consensus Statement of 1997. Med Clin North Am 1998; 82 (3): 613-33.

3. Guyatt GH, Sackett DL, Cook DJ. Users' guides to the medi- cal literature. II. How to use an article about therapy or prevention. B. What were the results and will they help me in caring for my patients? Evidence-Based Medicine Working Group. JAMA 1994; 271 (1): 59-63.

4. Kujath P, Spannagel U, Habscheid W. Incidence and Prophylaxis of Deep Venous Thrombosis in Outpatiens with Injury of the Lower Limb. Haemostassis 1993; 23 (Supl. 1): 20-6. 